\title{
Percepção da autoimagem corporal de universitários
}

\author{
Body self-image perception of university students
}

\author{
Priscila Evangelista Melo, ${ }^{1}$ Sérgio Cardoso Barcelos, ${ }^{2}$ Marina Garcia Manochio, ${ }^{3}$ \\ Helena Siqueira Vassimon, ${ }^{3}$ Cléria Maria Lobo Bittar ${ }^{3}$ \\ 'Universidade de Viçosa (UFV), Viçosa, MG, Brasil. \\ 2Centro Federal de Educação Tecnológica de Araxá, Araxá, MG, Brasil. \\ ¿nniversidade de Franca (Unifran), Franca, SP, Brasil.
}

Recebido em: 26/06/2016 / Aceito em: 10/08/2016 / Publicado em: 30/09/2016

profa.cleriabittar@gmail.com

\section{RESUMO}

Objetivo: o objetivo foi conhecer a percepção da autoimagem e satisfação corporal de universitários. Método: estudo quantitativo descritivo que utilizou dados de uma dissertação vinculada ao projeto Universidade Promotora de Saúde. Participaram 434 universitárias e 153 universitários, com idade de 18 a 30 anos que responderam dados sobre sexo, peso e altura, satisfação corporal e hábitos alimentares, além de se situarem, segundo a Escala de Silhuetas, dentro da plataforma Surveymonkey ${ }^{\circledR}$. Resultados: de acordo com o peso e estatura relatados, foi observado que $46,4 \%$ dos homens e $33,6 \%$ das mulheres eram considerados sobrepesos ou obesos. Em relação à Escala de Silhuetas foi possível observar que o desvio entre o IMC referido e o IMC percebido foi significativamente diferente para as mulheres $\left(-2,59 \mathrm{~kg} / \mathrm{m}^{2} \pm 2,36\right)$ para os homens $(-1,71$ $\mathrm{kg} / \mathrm{m}^{2} \pm 2,10$ ). Em ambos os grupos os indivíduos apresentavam uma distorção da imagem corporal, havendo uma percepção de estarem mais magros do que o real. Foi possível observar que as mulheres tinham maior distorção da imagem corporal que os homens $(p=0,01)$, e quanto à satisfação corporal elas se diziam pouco satisfeitas, enquanto os homens se mostraram bastante satisfeitos ( $p=0,03$ ). Também relataram se sentirem mais gordas quando comparadas a outras muIheres da mesma idade, o que não ocorreu com o grupo masculino. Universitários classificados como sobrepeso e/ou obeso se perceberam mais magros em ambos os sexos. Considerações finais: este trabalho reforça a necessidade de ações para maior conhecimento da percepção corporal por universitários, visando melhora na autoestima e qualidade de vida.

Palavras-chave: Autoimagem; autopercepção; universitários; universidade promotora de saúde.

\section{ABSTRACT}

Objective: was to know the perception of selfimage and body satisfaction of university students. Method: it is a descriptive quantitative study, in which data was used from a thesis linked to the project Health Promoter University. 434 female and 153 male university students between the ages of 18 to 30 years old participated, answering questions about gender, weight, height, body satisfaction and food habits, as well as identified themselves in the Scale Silhouettes within the Surveymonkey ${ }^{\circledR}$ platform. Results: according to the reported height and weight, it was observed that $46.4 \%$ of the men and $33.6 \%$ the women were considered overweight or obese. Regarding to the Scale Silhouettes it was observed a difference between the $B M I$ referred and the $B M I$ perceived, being significantly different for women $\left(-2.59 \mathrm{~kg} / \mathrm{m}^{2} \pm 2.36\right)$ and for men $(-1.71 \mathrm{~kg} / \mathrm{m} 2 \pm 10)$. In both groups, the individuals had a distortion of self body image, perceiving being slimmer than reality. It was observed that women had a higher self body image distortion than men $(p=$ 0.01), while on the self body satisfaction women also said they were little satisfied, whereof men were quite satisfied ( $p=0.03$ ). Furthermore women reported feeling fatter when compared to other women of the same age, what did not occur with the male group. Closing remarks: university students classified as overweight and / or obese perceived themselves thinner in both sexes. This study reinforces the need for actions to increase the understanding of self body awareness by university students, aiming to improve self-esteem and quality of life.

Keywords: Self Concept; self-perception; university students; health promoting university. 


\section{INTRODUÇÃO}

A universidade é um ambiente que pode desenvolver um papel importante na formação do profissional com consciência de atitudes saudáveis. É fato que os alunos passam grande parte do seu tempo nesse ambiente sem supervisão dos pais, o que pode deixá-los vulneráveis a condutas pouco saudáveis, como uso abusivo de bebidas, tabaco e drogas, predispondo-os a doenças. ${ }^{1,2}$

Silva $^{3}$ e Silva e Delorme ${ }^{4}$ citam o ambiente de aprendizagem como local onde se deve promover a articulação e empoderamento de alunos, comunidade e familiares. Nessa direção, foi concebida a ideia das Escolas Promotoras de Saúde (EPSs), proposta a partir da qual surgiram as Universidades Promotoras de Saúde (UPSs), que se baseiam nas cartas da promoção da saúde. ${ }^{5,6}$

As universidades promotoras de saúde atuam na formação de profissionais com princípios da promoção de saúde. $O$ envolvimento dessas instituições em ações saudáveis é interessante, pois, através destas, proporcionam melhora na qualidade de vida de seus alunos. As universidades envolvidas em programas de saúde têm sua imagem valorizada pela sociedade, além de assumir um papel de importância para a saúde local, regional e nacional. ${ }^{7-11}$

Nas últimas décadas, mudanças no estilo de vida, como a elevada ingestão de alimentos hipercalóricos, somadas à diminuição dos níveis de atividade física têm contribuído para o aumento do Índice de Massa Corporal (IMC), configurando sobrepeso e obesidade. Por outro lado, os padrões de beleza exigem corpos cada vez mais magros. Com isso, há uma crescente insatisfação da população em relação à autoimagem. ${ }^{12}$

A escala de figura de silhuetas tem sido bastante utilizada para avaliar a percepção da imagem corporal: trata-se de instrumento simples e de baixo custo. Além disso, as respostas demandam pouco tempo, o que facilita a sua aplicação. ${ }^{13-15}$ Por meio dessa escala, pode-se verificar se a percepção e satisfação são condizentes com o IMC.

Gardner et al. ${ }^{16}$ relatam alguns estudos epidemiológicos que vêm reunindo dados referentes à percepção da imagem corporal, medidas de peso e estatura referidos para predizer o IMC e estimar o estado nutricional da população o que tem possibilitado verificar uma tendência de indivíduos eutróficos de subestimar seu IMC, através da imagem corporal.

A percepção da imagem corporal sofre influências de componentes físicos, psicológicos, ambientais e comportamentais, ${ }^{17}$ tais como pensamentos, crenças, sentimentos e atitudes relacionadas ao próprio corpo. ${ }^{18}$ Quando essa percepção está subestimada ou superestimada, pode originar distúrbios psicológicos, transtornos alimentares, além de outros problemas relacionados ao ambiente social. ${ }^{19,17}$

Fatores como a influência da mídia e a prática de alguns esportes podem contribuir diretamente para a forma como o indivíduo se percebe. ${ }^{20}$

Os objetivos deste estudo foram conhecer a percepção da autoimagem e satisfação corporal de um grupo de universitários com o intuito de possibilitar a criação futura de ações efetivas promotoras de saúde, e verificar se havia diferença entre mulheres e homens em relação à percepção da autoimagem e satisfação corporal.

\section{MÉTODO E PROCEDIMENTOS}

Este estudo é de caráter descritivo e de abordagem quantitativa e faz parte do projeto de pesquisa sobre Universidades Promotoras de Saúde (UPSs), realizado por Petersen, ${ }^{1}$ que visou testar a aceitabilidade de um sistema de levantamento de informações sobre a saúde de universitários. O projeto seguiu as normas que regulamentam a pesquisa envolvendo seres humanos contidas na resolução CNS 466/12 do Conselho Nacional de Saúde, submetido ao Comitê de Ética e Pesquisa da UNIFRAN, e aprovado sob o protocolo de número 63/11.

$\mathrm{O}$ instrumento foi elaborado para a pesquisa na plataforma Surveymonkey ${ }^{\circledR}$ e disponibilizado através de um link pelo departamento de tecnologia de informação (TI) da Universidade no portal na área de serviços acadêmicos (ASA). O convite para participar da pesquisa ficou disponível para os alunos da graduação que acessaram o site da Universidade com senha e login pessoal durante o período de 3 a 17 de novembro de 2011, totalizando 15 dias. Após leitura do convite para participar da pesquisa os participantes tinham acesso ao termo de consentimento livre e esclarecido.

O questionário era composto de 25 questões que versavam sobre sexo, idade, etnia, curso e ano que está cursando, estado civil, período de estudo, filhos, moradia, procedência, trabalho, religião, nível socioeconômico e hobbies. Após responderem a essas questões, o respondente escolhia um tema que desejava responder e era direcionado para um dos seguintes questionários: 1) bem-estar subjetivo, satisfação com a vida e percepção de estresse; 2) saúde em geral, qualidade de vida, autoimagem e alimentação; 3) vida afetiva e sexual, comportamentos de risco e percepção do ambiente universitário; 4) uso de bebidas alcoólicas, tabaco, drogas ilegais e substâncias psicoativas.

Para a análise dos resultados deste estudo, foram considerados apenas a amostra que respondeu ao tema saúde em geral, qualidade de vida, autoimagem $e$ alimentação. Para este eixo temático, obteve-se a resposta de 660 universitários.

Além dos dados sobre sexo e idade, foram consideradas variáveis de peso e estatura relatadas além do uso dos resultados da escala de figura de silhuetas construída para a população brasileira. A escala apresenta figuras de corpos em diferentes tamanhos, com nove imagens, representadas em sequência, desde a silhueta mais magra até a mais gorda. Os participantes escolheram um cartão, dentre os dispostos em série ordenada ascendente, com a silhueta que mais se aproximava de seu próprio corpo, gerando, assim, o respectivo IMC Atual. ${ }^{21}$

Adotou-se a equação do valor de IMC referente à imagem na escala de figura de silhuetas. Este foi denominado "IMC Real/Referido" (IMCR), uma vez que não foi feita a aferição direta de peso/ estatura dos participantes, mas sim a resposta dada por ele. Para avaliar a acurácia da imagem corporal, foi calculada a diferença entre as médias de IMC Real/Referido e Atual (IMC (R-A), sendo que resultados próximos de zero indi- 
cam percepção acurada da própria imagem. Resultados negativos apontam subestimação do tamanho corporal, enquanto os positivos indicam sua superestimação. ${ }^{21}$

$\mathrm{O}$ teste de qui-quadrado foi utilizado para verificar associação entre as variáveis: "percepção da imagem corporal" e "sexo" ou "classificação nutricional". Para análise dos dados referentes à inacurácia da percepção da imagem, primeiramente, foi realizado teste de normalidade, em que as variáveis contínuas foram apresentadas em média e desvio-padrão. Para comparação entre variáveis contínuas em mais de três grupos foi utilizado o teste de normalidade Shapiro-Wilk e análise de resíduos. Considerando a distribuição normal, foi aplicado teste de análise de variância (ANOVA) dois fatores e considerado nível de significância de $5 \% .{ }^{22}$

\section{RESULTADOS E DISCUSSÃO}

O estudo avaliou 660 questionários respondidos sobre autoimagem corporal e alimentação. Foi necessário excluir $73(11 \%)$ questionários incompletos, totalizando uma amostra de 587 universitários, sendo que 434 (73,9\%) eram mulheres e 153 (26,1\%) eram homens. Quanto à distribuição por faixa etária, 300 alunos (51,1\%) tinham entre 18 e 23 anos, 123 (21\%) entre 24 e 29 e 164 (27\%) tinham 30 anos ou mais.

$\mathrm{Na}$ Tabela 1 foram expostos os resultados referentes à classificação nutricional por sexo, segundo os dados sobre peso e estatura informados pelo participante, IMC Real/Referido" (IMCR). Ao considerar a somatória dos universitários com diagnósticos de sobrepeso e obesidade, houve diferença entre o sexo. Observou-se prevalência de $46,4 \%$ entre os homens e 33,6\% entre as mulheres $(p=0,02)$.

Em relação à percepção da autoimagem corporal, os dados foram apresentados na Tabela 2. De acordo com os resultados, pode-se considerar que houve diferença significativa na acurácia da percepção da imagem corporal entre os sexos dos universitários classificados com desnutrição ou obesidade $(p=0,01)$. Entre os universitários classificados com desnutrição, tanto nas mulheres, quanto nos homens, o IMC Real/Referido foi inferior ao IMC Atual, ou seja, os participantes se acham maiores do que realmente são, demonstrando superestimação da percepção corporal, as mulheres em média $2,7 \mathrm{~kg} / \mathrm{m}^{2}$ com desvio-padrão de 0,84 , e os homens em média com $3,8 \mathrm{~kg} / \mathrm{m}^{2} \mathrm{com}$ desvio-padrão de 2,25 . Nos indivíduos com obesidade o IMC Real/Referido foi superior ao IMC Atual, indicando subestimação da percepção corporal, maior nas mulheres $\left(-2,6 \mathrm{~kg} / \mathrm{m}^{2}\right)$ em relação aos homens $\left(-1,7 \mathrm{~kg} / \mathrm{m}^{2}\right)$.

Foi possível observar que, independentemente do sexo, os universitários deste estudo com classificação nutricional de desnutrição se percebem maiores do que realmente são, e aqueles com obesidade se perceberam mais magros do que relataram. Diferente do estudo de
Tabela 2 - Caracterização da diferença entre IMC Real/ Referido e Atual pela escala de figura de silhuetas de acordo com sexo e classificação nutricional de um grupo de universitários.

\begin{tabular}{lcccc}
\hline Sexo & Classe & IMC & IMCA & IMC (A-R) \\
\hline Masculino & Desnutrição & 17,7 & 21,6 & $3,8 \mathrm{~b}$ \\
& D.P & 0,76 & 2,29 & 2,25 \\
& Eutrófico & 22,3 & 24,2 & 1,9 \\
& DP & 1,8 & 2,9 & 3,08 \\
& Sobrepeso & 26,6 & 27,7 & 1,1 \\
& DP & 1,36 & 2,17 & 2,0 \\
& Obeso & 32,8 & 31,1 & $-1,7$ \\
& DP & 2,59 & 2,75 & 2,1 \\
& & & & \\
& Desnutrição & 17,3 & 20 & $2,7^{\text {a }}$ \\
& D.P & 1,04 & 0,65 & 0,84 \\
& Eutrófico & 21,7 & 23,7 & 2,0 \\
& DP & 1,74 & 2,31 & 1,98 \\
& Sobrepeso & 26,9 & 27,4 & 0,6 \\
& DP & 1,37 & 2,06 & 1,8 \\
& Obeso & 32,8 & 30,2 & $-2,6$ \\
& DP & 2,11 & 2,28 & 2,36 \\
\hline
\end{tabular}

Legenda: $a \neq b(p=0,02)$ para sexo e classificação de IMC (índice de massa corporal) pelo teste de qui-quadrado.

Kakeshita e Almeida ${ }^{23}$ que, ao avaliarem a relação entre IMC e a percepção da autoimagem em 106 universitários, perceberam que as mulheres apresentaram maior tendência em inacurácia da percepção da autoimagem.

No presente estudo, quanto à satisfação corporal, foi possível observar que os homens demonstraram estar mais satisfeitos com seus corpos, do que as muIheres $(p=0,03)$.

Graup et al. ${ }^{17}$ observaram resultados semelhantes de maior insatisfação corporal entre os indivíduos do sexo feminino.

Quando analisados os universitários do presente estudo que foram classificados com sobrepeso, cerca de $34 \%$ dos homens demonstraram-se "bastante" satisfeitos e 39\% das mulheres "pouco" satisfeitas, e dentre os classificados com obesidade $47 \%$ das mulheres declararam estarem "pouco" satisfeitas. Quando analisados os universitários classificados com sobrepeso, cerca de $34 \%$ dos homens demonstraram-se "bastante" satisfeitos e $39 \%$ das mulheres "pouco" satisfeitas, e dentre os classificados com obesidade $47 \%$ das mulheres declararam estarem "pouco" satisfeitas. As mulheres com sobrepeso e obesidade estão mais insatisfeitas com o corpo do que os homens, já que as variáveis "bastante" satisfeitas foram mais frequentes para a resposta dos homens e a variável "pouco" satisfeito mais frequente entre as mulheres $(p<0,05)$.

Segundo Damasceno et al., ${ }^{24}$ as mulheres e os homens buscam o corpo perfeito, sendo que as mulheres almejam corpos mais magros e os homens corpos mais fortes e volumosos.

Tabela 1 - Distribuição do estado nutricional, segundo IMC de grupo de universitários (IMCR).

\begin{tabular}{lccccc}
\hline Sexo & Desnutrido N (\%) & Eutrófico N (\%) & Sobrepeso N (\%) & Obeso N (\%) \\
\hline Masculino & $8(5,2)$ & $74(48,4)$ & $47(30,7)^{\mathrm{a}}$ & $24(15,7)$ & Total N (\%) \\
Feminino & $36(8,3)$ & $252(58,1)$ & $87(20,0)^{\mathrm{b}}$ & $59(13,6)$ & $43(100)$ \\
TOTAL & $44(7,5)$ & $326(55,5)$ & $134(22,8)$ & $83(14,4)$ & $587(100)$ \\
\hline
\end{tabular}

Legenda: $a \neq b(p=0,02)$ para sexo e classificação de IMC (índice de massa corporal) pelo teste de qui-quadrado. 
Houve diferença significativa entre os universitários avaliados no presente estudo para o sexo feminino e masculino $(p=0,05)$ no total da amostra, referente à comparação da percepção do indivíduo em relação à outra pessoa de sua idade. Verificou-se que as mulheres se consideravam mais gordas do que outra pessoa de sua idade, diferente dos homens, que se percebiam "nem gordo nem magro". Quando comparados os participantes com sobrepeso de ambos os grupos, as mulheres se percebiam mais gordas do que os homens. Como visto nos resultados, observou-se que tanto os homens como as mulheres classificados com obesidade escolheram na escala de figura de silhuetas inferiores à imagem referente ao IMCR. Portanto, os indivíduos desta amostra parecem não se perceber acima do peso, o que pode ser um fator relevante para o agravamento da obesidade.

Antunes, Pozzobon e Pereira ${ }^{25}$ avaliaram, através da escala de figura de silhuetas, a percepção da imagem corporal e perfil nutricional em 30 mulheres. Os resultados apontaram que cerca de 93,3\% das mulheres escolheram a imagem de uma silhueta inferior à que realmente possuem.

Branco, Hilário e Cintra ${ }^{26}$ verificaram em estudo sobre relação do estado nutricional com a percepção e satisfação da imagem corporal de adolescente, que indivíduos do sexo masculino se percebiam em IMC abaixo do atual. É provável que, quando o indivíduo apresenta inacurácia da percepção da imagem, conforme demonstrado no presente estudo e em outros estudos ele se torne vulnerável aos problemas do excesso de peso.

Bosi et al. ${ }^{27}$ (p. 111) defendem que "os valores do IMC informado e o desejado tendem, ambos, para o limite inferior da normalidade, o que sugere um desejo de adequação ao padrão ideal de corpo na atualidade".

No que diz respeito ao nível de satisfação com o corpo, as mulheres demonstraram maior insatisfação do que os homens, principalmente entre os universitários classificados com sobrepeso.

Rech et al. ${ }^{28}$ conduziram um estudo sobre autopercepção de imagem corporal em um grupo de universitários, e verificaram que a insatisfação com o corpo entre os homens e as mulheres não diferia.

Os problemas relacionados com a insatisfação da imagem corporal podem desencadear problemas a longo prazo na vida do indivíduo. Saikali et al. ${ }^{29}$ enfatizam questão do desenvolvimento de depressão e transtornos alimentares, exclusão, dentre outros distúrbios relacionados com a autoimagem em detrimento do excesso de peso.

A obesidade traz prejuízos emocionais devido à não aceitação deste indivíduo pela sociedade por não estar enquadrado nos padrões de beleza predefinidos ${ }^{30}$, podendo, ainda, estar relacionado com a insatisfação da autoimagem corporal e, consequentemente, pode acarretar problemas relacionado à autoestima.

Foi notada no presente estudo a insatisfação com a autoimagem entre os homens e as mulheres, porém mais expressiva entre o sexo feminino. Este fenômeno de insatisfação se dá entre o sexo feminino, possivelmente, devido ao desejo de emagrecer com o aumento da idade e contrariamente, no sexo masculino, prevalece o desejo de ganhar peso num porte atlético. ${ }^{31}$

Igualmente Kakeshita e Almeida ${ }^{23}$ - em seu estudo com universitários - verificaram que as "mulheres aumentaram a sua insatisfação com a imagem corporal de forma diretamente proporcional ao aumento da classificação nutricional a que pertenciam" (p. 5). Assim como Oliveira et $\mathrm{al}^{32}$, que encontraram resultados semelhantes em seu estudo e alertam que a insatisfação com o corpo entre mulheres jovens pode estar relacionada ao desenvolvimento de distúrbios alimentares.

A insatisfação com a imagem corporal pode afetar a autoestima do indivíduo, e quando isso acontece impede o desenvolvimento de uma vida social, levando-o muitas vezes à depressão, além de outros distúrbios. ${ }^{33,30}$

Segundo relatado por Souto e Ferro-Burcher ${ }^{34}$ o distúrbio alimentar é comum em mulheres que apresentam desejo de perder peso devido à insatisfação com o corpo. $\mathrm{O}$ estudo de Nunes et al. ${ }^{35}$ demonstra que entre as mulheres a insatisfação corporal também pode levar a comportamento alimentar de risco ou anormal.

A imagem e a identidade corporal são um conjunto de significados e experiências, vivenciados ao longo da vida, a partir dos quais o ser humano conceitua seu próprio corpo. São influenciadas por fatores sociais, culturais e psicodinâmicos. ${ }^{36}$ Tal processo de formação da imagem corporal inicia-se na infância, por volta dos dois anos de idade, e se modifica no decorrer dos anos. Ocorre a partir da descoberta sobre o que é socialmente atraente e não atraente, e isso certamente traz consequências sentimentais relacionadas à autovalorização. ${ }^{37}$ Contudo é na adolescência, em meio às transformações hormonais, funcionais, afetivas e sociais, que as mudanças corporais adquirem importância, pois é por meio de seu corpo que o jovem pode melhor se perceber. Frequentemente, porém, ocorrem distorções da realidade, visto que a imagem corporal pode não corresponder à imagem real. ${ }^{38}$

Nessa linha de pensamento, Heatherton e Wyland ${ }^{39}$ defendem que a forma como nos percebemos é influenciada pela maneira como os outros nos veem.

A inacurácia da distorção da autoimagem corporal pode ser componente não só para detectar indivíduos subnutridos, que se percebem como sobrepesos, mas também o contrário, indivíduos obesos que se identificam como eutróficos. ${ }^{23}$

Conforme apresentado nos resultados deste estudo, os homens demonstram estar mais satisfeitos com o corpo do que as mulheres. Este fenômeno pode ser compreendido, já que os homens por sua vez tendem a querer corpos mais volumosos e as mulheres apresentam desejo de ter corpos mais magros e menos volumosos. ${ }^{24,30}$

Portanto, é possível inferir que talvez esse desejo possa interferir no ganho de peso, tornando-os mais vulneráveis às DCNTs por não perceberem o excesso de peso.

Como visto, o problema do sobrepeso e obesidade interfere na percepção e satisfação com a autoimagem corporal. Portanto, é possível inferir que a luta contra o excesso de peso deve agrupar diversos saberes e práticas profissionais, considerando os aspectos multifatoriais desta DCNT.

Pode-se pensar como estratégia a possibilidade de a universidade promover um ambiente favorável a bons hábitos alimentares, contribuindo paralelamente no controle do peso dos universitários. 
Ações conjuntas com a sociedade escolar são necessárias na realização de projetos para formação de ambientes saudáveis, ${ }^{40,41}$ já que o ambiente universitário é um local de aprendizado, portanto, propício à promoção de saúde.

Ressalta-se a possibilidade de haver um viés para este resultado no presente estudo, pois ao referir o peso e altura, o respondente pode ter informado valores diferentes do real. Cabe dizer, porém, que a utilização de peso e estatura autorreferidos foi validada em estudos com diferentes faixas etárias. ${ }^{42,43}$

\section{CONSIDERACִÕES FINAIS}

A universidade possui um papel importante na formação do indivíduo, tanto como profissional, como membro de uma sociedade, pois o ambiente universitário pode promover benefícios principalmente na promoção da saúde mental e psicológica desse jovem universitário. Desta forma torna-se importante considerar relevância da universidade conhecer o nível de saúde do seu aluno, subsidiando ações para uma educação saudável.

Os resultados do presente estudo evidenciam a inacurácia da percepção da autoimagem que podem afetar a saúde e qualidade de vida do indivíduo. Portanto, o estudo alerta sobre a importância de ações de promoção de saúde no ambiente universitário, considerando as questões acerca da acurácia da percepção e satisfação corporal, considerando as diferenças entre os sexos e a importância atribuída à alimentação e as percepções do peso e da imagem corporal.

\section{REFERÊNCIAS}

1. Petersen CB. Análise do processo de implantação de pesquisa online para levantamento de dados em saúde junto a universitários. 2012. 171f. Dissertação (Mestrado em Promoção da Saúde) - Universidade de Franca, Franca.

2. Franca C, Colares V. Estudo comparativo de condutas de saúde entre universitários no início e no final do curso. Rev Saúde Pública 2008;42(3):420-7.

3. Silva C S. Escola Promotora de Saúde: uma visão crítica da saúde escolar. In: Harada, J. et al. Caderno de Escolas Promotoras de Saúde. In: Sociedade Brasileira de PediatriaDepartamento Científico de Saúde Escolar. 2006, p. 14-20.

4. Silva CS, Delorme MIC. Apresentação das experiências. In: BRASIL. Ministério da Saúde. Escolas promotoras de saúde: experiências do Brasil. Ministério da Saúde, Organização Pan-Americana da Saúde. Brasília: Ministério da Saúde, 2007. p. 304.

5. Brasil. Ministério da Saúde. Projeto de Promoção da Saúde. As cartas da Promoção à Saúde/Ministério da Saúde, Secretaria de Políticas da Saúde, Projeto Promoção de Saúde. Brasília: Ministério da Saúde, 2002.

6. OMS. Organização Mundial da Saúde. Escolas Promotoras da Saúde: modelo e guia para ação. Washington DC: Opas, 2003.

7. Mello ALSF de, Moyses ST, Moyses SJ. A universidade promotora de saúde e as mudanças na formação profissional. Interface (Botucatu) 2010;14(34):683-92.

8. Ippolito-Shepherd Jl. A promoção da saúde no âmbito escolar: a iniciativa regional escolas promotoras de saúde. In: Escolas promotoras de saúde: manual da Sociedade
Brasileira de Pediatria. [s.l. : s.n.], 2003. p. 8-13.

9. Loureiro I. A importância da educação alimentar: o papel das escolas promotoras de saúde. Revista de Educação Nutricional 2004;22(2):43-55.

10. Precioso J. Educação para saúde na universidade: um estudo realizado em alunos da Universidade do Minho. Revista Eletrônica de Enseñanza de las Ciencias 2004;3(2):161-70.

11. Schmitz BAS, Recine E, Cardosos GT, Silva JRM, Amorim NFA, Bernardon R, Rodrigues MLCF. A escola promovendo hábitos alimentares saudáveis: uma proposta metodológica de capacitação para educadores e donos de cantina escolar. Cad Saúde Pública 2008;24(S3):12-22.

12. Andrade A, Bosi MLM. Mídia e subjetividade: impacto no comportamento alimentar feminino. Rev Nutr 2003;16(1):117-25.

13. Cash TF, Pruzinsky T. (Eds) Body Image: a handbook of theory, research, and clinical practice. New York: Guilford Press, 2002.

14. Gardner RM. Body Image Assessment of Children. In: Cash, TF.; Prusinky, T. Body Image: a handbook of theory, research, and clinical practice. New York: Guilford Press 2002;15:127-34.

15. Hart EA. Avaliando a imagem corporal. In: Tritschler K. Medidas e avaliação em educação física e esportes de Barrow \& MCGEe. São Paulo: Manole 2003;11:457-88.

16. Gardner RM, Stark K, Jackson NA, Friedman BN. Development and validation of two new scales for assessment of body image. Perceptual Motor Skills 1999;89(3):981-93.

17. Graup S, Pereira EF, Lopes AS, Araújo VC, Legnani RFS, Borgatto AF. Associação entre a percepção da imagem corporal e indicadores antropométricos de escolares. RBEFE 2008;22(2):129-38.

18. Cash T F. Body image: past, present, and future. Bory image 2004;1:1-5.

19. Fontes M. Uma leitura do culto contemporâneo ao corpo. Contemporânea, Salvador 2006;4(1):117-36.

20. Paulucci EZC, Ferreira A. Corpo e imagem: relações entre medidas antropométricas e aceitação pessoas da imagem corporal em praticantes de jazz de competição. Refeld 2009;4(2):53-61.

21. Kakeshita IS. Estudo das relações sociais o estado nutricional, a percepção da imagem corporal e o comportamento alimentar em adulto. 2004. 73f. Dissertação (Mestrado em Psicobiologia) - Faculdade de Filosofia Ciências e Letras de Ribeirão Preto- USP.

22. Anjos A. dos. Análise de Variância. 2008. Disponível em: <http://www.est.ufpr.br/ce003/ material/cap7.pdf>.

23. Kakeshita IS.; Almeida, S. de S. Relação entre índice de massa corporal e a percepção da autoimagem em universitários. Rev Saúde Pública 2006;40(3):497-504.

24. Damasceno VO, Lima JRP, Vianna JM, Vianna VRA, Novaes JS. Tipo físico ideal e satisfação com a imagem corporal de praticantes de caminhada. Rev Bras Med Esporte 2005;11(3):181-6.

25. Antunes AV, Pozzobon A, Pereira ALB. Avaliação antropométrica, autopercepção corporal e perfil nutricional de mulheres adultas. Revista Destaques Acadêmico 2011;3(3).

26. Branco LM, Hilario MOE, Cintra I de P. Percepção e satisfação corporal em adolescentes e a relação com seu estado nutricional. Rev Psiquiatr Clín 2006;33(6):292-6.

27. Bosi MLM, Luiz RR, Morgado CMC, Costa MLS, Carvalho RJ. Autopercepção da imagem corporal entre estudantes de nutrição: um estudo no município do Rio de Janeiro. Jornal Brasileiro de Psiquiatria 2006;55(2):108-13.

28. Rech CR, Araújo EDS, Vanat JR. Autopercepção da imagem 
corporal em estudantes do curso de educação física. Rev Bras Educ Fís Esporte (Impr.) 2010;24(2):285-92. doi: 10.1590/S1807-55092010000200011

29. Saikali CJ, Scalfaro BM; Cordás TA. Imagem corporal nos transtornos alimentares. Rev Psiquiatr Clín 2004;31(4):164-6.

30. Serra GMA, Santos EM. Saúde e Mídia na construção da obesidade e do corpo perfeito. Ciênc Saúde Coletiva 2003;8(3):691-701.

31. Vilela JEM, Lamounier JÁ, Oliveira RG, Ribeiro RQC, Gomes ELC, Barros Neto JR. Avaliação do comportamento alimentar em crianças e adolescentes de Belo Horizonte. Psiquiatr Biol 2001;9,(3):121-30.

32. Oliveira FP, Bosi MLM, Vigário PS, Vieira RS. Comportamento alimentar e imagem corporal em atletas. Rev Bras Med Esporte 2003;9(6):348-56.

33. Menossi MJ, Lima RAG. A problemática do sofrimento: percepção do adolescente com câncer. Revista da Escola de Enfermagem - USP 2000;34(1):45-51.

34. Souto S, Ferro-Bucher JSN. Práticas indiscriminadas de dietas de emagrecimento e o desenvolvimento de transtornos alimentares. Rev Nutr 2006;19(6):693-704. doi: 10.1590/ S1415-52732006000600006.

35. Nunes MA, Olinto MTA, Barros FC, Camey S. Influência da percepção do peso e do índice de massa corporal nos comportamentos alimentares anormais. Rev Bras Psiquiatr 2001;23(1):21-7.

36. Tavares MCGCF. Imagem corporal: conceito e desenvolvimento. Barueri, SP: Manole, 2003.

37. Braggion GF, Matsudo SMM, Matsudo VKR. Consumo alimentar, atividade física e percepção da aparência corporal em adolescentes. Rev Bras Ciên Mov 2000;8(1):15-21.

38. Castilho SM. A imagem corporal. Santo André: ESETec Ed. Associados, 2001. p. 40-6.

39. Heatherton TF, Wyland CL. Assessing Self-esteem. In: Dans SJ, Lopez CR, Snyder (Eds), Positive psychological assessment: a handbook of models and measures. Washington, DC: American Psychological Association, 2003. p. 219-34.

40. Shi-Chang $X$, Xin-Wei $Z$, Shui-Yang $X$, Shu-Ming T, SenHai Y, Aldinger C, Glasauer P. Creating health-promoting schools in China with a focus on nutrition. Health Promotion International 2004;19(4):409-18.

41. Perry C, Bishop DB, Taylor GL, Davis M, Story M, Gray C, Bishop SC, Mays RA, Lytle LA, Harnack L. A randomized school trial of environmental strategies to encourage fruit and vegetable consumption among children. Health Educ Behav 2004;31: 65-76.

42. Silveira EA, Araújo CL, Gigante DP, Barros AJD, Lima MS. Validação do peso e altura referidos para o diagnóstico do estado nutricional em uma população de adultos no Sul do Brasil. Cad Saúde Pública 2005;21(1):235-45. doi: 10.1590/ S0102-311X2005000100026.

43. Rech CR, Petroski EL, Böing O, Babel Junior RJ, Soares MR. Concordância entre as medidas de peso e estatura mensuradas e autorreferidas para o diagnóstico do estado nutricional de idosos residentes no sul do Brasil. Rev Bras Med Esporte 2008;14(2):126-31.

Como citar: MELO, Priscila Evangelista et al. Percepção da autoimagem corporal de universitários: uma possibilidade de atuação na universidade promotora de saúde. Cinergis, Santa Cruz do Sul, v. 17, n. 3, set. 2016. ISSN 2177-4005. Disponível em: <https://online.unisc.br/seer/index.php/cinergis/article/view/7760>. Acesso em: 11 out. 2016. doi:http://dx.doi. org/10.17058/cinergis. v17i3.7760. 